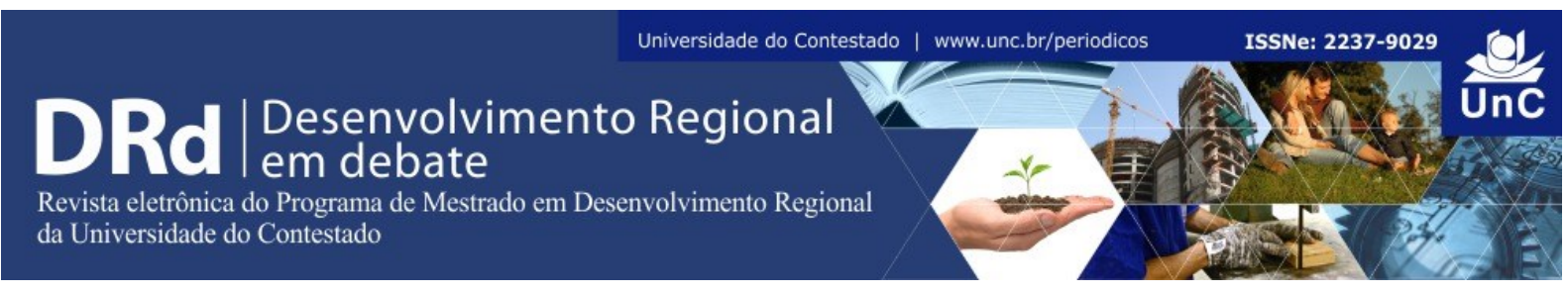

\title{
ANÁLISE ESPACIAL DA TAXA DE COBERTURA DO PROGRAMA BOLSA FAMÍLIA PARA OS MUNICÍPIOS BRASILEIROS
}

\author{
Jéssica Faciroli ${ }^{1}$ \\ Tássio Ferenzini Martins Sirqueira ${ }^{2}$
}

\begin{abstract}
RESUMO
Este trabalho propõe verificar a existência da associação espacial entre a taxa de cobertura do programa Bolsa Família e a taxa de pobreza para os municípios brasileiros, com o objetivo de verificar se a taxa de cobertura do programa está focalizada nas regiões com maiores taxas de pobreza. A metodologia utilizada consistiu na análise explanatória dos dados espaciais para as variáveis taxa de pobreza e taxa de cobertura do programa. Para as estimações através dos modelos MQO, SAR e SEM utilizou-se as variáveis taxa de pobreza, taxa de cobertura, número de CRAS, valor total repassado do programa para os municípios, Índice de Desenvolvimento Humano, acompanhamento da saúde e da educação dos beneficiários. Os resultados apontaram para a existência de autocorrelação positiva entre a taxa de cobertura do programa Bolsa Família e a taxa de pobreza.
\end{abstract}

Palavras-chave: Bolsa Família. Pobreza. Análise Espacial.

\section{SPATIAL ANALYSIS OF THE COVERAGE RATE OF THE BOLSA FAMILIA PROGRAM FOR THE BRAZILIAN MUNICIPALITIES}

\begin{abstract}
The paper proposes to verify the existence of the spatial association between the coverage rate of the Bolsa Familia program and the poverty rate for the municipalities of Brazil, in order to verify if the coverage rate of the program is focused on the regions with the highest poverty rates. The methodology used consisted of the explanatory analysis of spatial data for the variables poverty rate and program coverage rate. For the estimations using the MQO, SAR and SEM models, the variables poverty rate, coverage rate, number of CRAS, total value transferred from the program to the municipality, human development index, health monitoring and beneficiaries' education were used. The results pointed to the existence of a positive autocorrelation between the coverage rate of the Bolsa Familia program and the poverty rate.
\end{abstract}

Keywords: Bolsa Familia. Poverty. Spatial Analysis.

${ }^{1}$ Graduada em Ciências Econômicas pela Universidade Federal de Juiz de Fora (2015) e mestranda em Economia Aplicada pela Universidade Federal de Juiz de Fora. Juiz de Fora. Minas Gerais. Brasil. E-mail:

jessicafacirolimb@gmail.com

${ }^{2}$ Graduado em Sistemas de Informação pelo Centro de Ensino Superior de Juiz de Fora (2013), mestrado em Ciência da Computação pela Universidade Federal de Juiz de Fora (2016) e doutorando em Informática pela Pontifícia Universidade Católica do Rio de Janeiro. É professor Assistente no Instituto Vianna Júnior. Juiz de Fora. Minas Gerais. Brasil. E-mail: tmsirqueira@vianna.edu.br

DRd - Desenvolvimento Regional em debate (ISSNe 2237-9029)

v. 7, n. 2, p. 118-137, jul./dez. 2017. 


\section{INTRODUÇÃO}

Segundo Ministério do Desenvolvimento Social (MDS) ${ }^{3}$, o objetivo do Programa Bolsa Família (PBF) é contribuir para superação da pobreza no país em três eixos de atuações: i) transferência de renda direta as famílias, que permitem alívio imediato da situação de pobreza; ii) ampliação do acesso a serviços públicos que apresentam direitos básicos nas áreas de saúde, educação e assistência social; para que as famílias rompam o ciclo intergeracional de reprodução da pobreza; iii) coordenação com outras ações e com outros programas do governo, de modo a apoiar as famílias para que superem a situação de vulnerabilidade e pobreza.

Com base nisso, a focalização do programa Bolsa Família consiste em priorizar os benefícios para as famílias que vivem em situação de extrema pobreza e pobreza. Essa identificação é feita através do Cadastro Único (CadÚnico), tendo como objetivo identificar e caracterizar famílias que vivem com renda mensal de até meio salário mínimo. Pelo Cadastro Único é possível analisar informações sobre os domicílios das famílias, acesso aos serviços públicos como saúde e educação, número de componentes nas famílias, dentre outras informações.

A seleção das famílias para o programa Bolsa Família é realizada através do MDS, com base nos dados do Cadastro Único, sendo a seleção feita a partir de cotas de benefícios municipais, previamente estabelecido com base no número de famílias que vivem em situação de pobreza em cada município. Caso o número de famílias cadastradas para o programa esteja acima do que foi estimado para o munícipio, a concessão deve priorizar as famílias com menor renda per capita e com maior número de crianças na faixa etária de 0 a 16 anos (CAMARGO et al., 2013).

Visto isso, um programa é considerado perfeitamente focalizado se transferir recursos para todas as pessoas elegíveis, redirecionando recursos para determinados grupos, considerados mais vulneráveis no conjunto da sociedade (KERSTENETZKY, 2009). Para Barros et al. (2010), a focalização do Programa Bolsa Família é elevada nos seguintes casos: i) se as famílias inseridas no Cadastro Único forem as mais pobres; ii) as informações coletadas forem de alta qualidade; iii) a metodologia para utilização de tais informações na seleção dos beneficiários for a mais adequada e iv) as cotas estabelecidas para cada município forem às próximas ao efetivo número de pobres no local.

Partindo desses princípios, este estudo tem o objetivo de analisar se a taxa de cobertura do programa Bolsa Família está elevada em municípios que apresentam altas taxas de pobreza, ou seja, se o programa está de fato atendendo as famílias que vivem em situação de pobreza. A taxa de cobertura do programa é uma variável que expressa o número de famílias que vivem em situação de pobreza numa dada região, e quantas deste total estão de fato recebendo o benefício do programa. É de esperar que regiões que têm elevada concentração de famílias vivendo em situação de pobreza apresentem maiores taxas de cobertura do programa.

${ }^{3}$ https://mds.gov.br/assuntos/bolsa-familia/o-que-e/acesso-a-educacao-e-saude 
O trabalho está organizado em quatro seções, além dessa introdução. Na segunda seção será discutido o programa Bolsa Família. Na terceira seção será apresentado o referencial teórico. Já na quarta seção discutiremos a metodologia usada. Pôr fim, na seção cinco têm-se os resultados e os trabalhos futuros.

\section{PROGRAMA BOLSA FAMÍLIA}

O programa Bolsa Família foi criado em outubro de 2003 por meio da medida provisória número 132, convertida posteriormente na lei número 10.836 em janeiro 2004 . Esse programa foi criado com a finalidade de unificar os programas preexistentes Bolsa Escola, Bolsa Alimentação, Auxílio-Gás e Fome Zero. O programa Bolsa Família consiste na transferência de renda direta para as famílias que vivem em situação de pobreza e extrema pobreza.

Para uma família realizar o cadastro no programa Bolsa Família, deve primeiramente se inscrever no CadÚnico. As famílias realizam tal cadastro no setor do Bolsa Família ou procuram os Centros de Referência da Assistência Social (CRAS) de cada cidade. O MDS oferece às prefeituras um formulário padrão, para coletar as informações das famílias elegíveis para o programa, sendo preenchido pelo município é enviado para base de dados da Caixa Econômica Federal (CEF), onde o MDS determina quais famílias serão beneficiadas e fornece a lista dos beneficiários à $\mathrm{CEF}$, que por sua vez efetua o depósito na conta dos beneficiários. Assim, as famílias beneficiadas recebem um cartão de saque, denominadas Cartão Bolsa Família (UNIÃO, 2009).

Cada município possui cotas de beneficiários baseadas nas estimativas de famílias em situação de pobreza. Logo, a concessão do benefício depende da quantidade de famílias atendidas no município, em relação às estimativas para essa localidade. Essa ação ocorre como uma forma do governo federal respeitar o limite orçamentário do programa. Segundo $\mathrm{MDS}^{4}$, programa trabalha com os seguintes benefícios: i) benefício básico (BB), ii) benefício variável $(\mathrm{BV})$, iii) benefício variável vinculado ao adolescente (BVA), iv) benefício para superação de extrema pobreza (BSP). Devido esta combinação de benefícios, cada família recebe um valor diferente, dependendo da sua renda mensal por pessoa e quantidade de membros na família.

O benefício básico é concedido às famílias que vivem em situação de extrema pobreza, mesmo que não tenham crianças, adolescentes, gestantes e nutrizes em sua composição, seu valor é de $\mathrm{R} \$ 85,00$ e a família deve ter renda per capita de até $\mathrm{R} \$ 85,00$. O benefício variável é concedido àquelas famílias em situação de pobreza e extrema pobreza, que tenham renda per capita de $\mathrm{R} \$ 170,00$ e apresentem em sua composição gestante, nutriz ou crianças e adolescentes de até 15 anos de idade. O valor deste benefício é $\mathrm{R} \$ 85,00$ mais $\mathrm{R} \$ 39,00$ se na família houver em sua composição um dos casos supracitado.

Já o benefício para superação da extrema pobreza, é concedido às famílias que se mantenham uma renda familiar por pessoa igual ou inferior a $\mathrm{R} \$ 85,00$ mesmo após receberem

\footnotetext{
${ }^{4}$ https://mds.gov.br/assuntos/bolsa-familia/o-que-e/beneficios 
os outros tipos de benefícios do programa. O valor do benefício é calculado caso a caso, de acordo com renda e quantidade de pessoas na família, para garantir que a família ultrapasse o piso de $\mathrm{R} \$ 85,00$ de renda por pessoa.

A família ao receber o benefício do programa Bolsa Família deve assumir alguns compromissos, chamados de condicionalidades. Não apenas os beneficiários que têm as condicionalidades, mas também o poder público deve ter foco nessas famílias. As condicionalidades são: i) na área de educação os responsáveis devem matricular as crianças e os adolescentes de 6 a 17 anos na escola e a frequência escolar deve ser de, pelo menos, 85\% nas aulas para crianças e adolescentes de 6 a 15 anos e de $75 \%$ para adolescentes de 16 e 17 anos, todo mês; ii) na área da saúde os responsáveis devem levar as crianças menores de sete anos para tomar as vacinas recomendadas pelas equipes de saúde e para pesar, medir e fazer acompanhamento do crescimento e desenvolvimento; iii) as gestantes devem fazer o pré-natal e ir às consultas nas Unidades de Saúde. Caso a família beneficiária descumpre os compromissos em um período de seis meses, ocorre o bloqueio e a impede de receber o benefício por um mês $\left(\mathrm{MDS}^{2}\right)$. Caso após o bloqueio houver novamente o descumprimento dos compromissos, o benefício fica suspenso por dois meses, sem possibilidade de rever as parcelas.

Os três níveis do governo devem trabalhar em conjunto para acompanhar as condicionalidades do programa, onde o poder público fica com o papel de garantir serviços de educação e saúde à população em situação de vulnerabilidade, identificar as famílias que estão com dificuldades e encaminha-las para rede de assistência social.

\section{REFERENCIAL TEÓRICO}

Entre os trabalhos que se propõem em investigar sobre o PBF e que contribuem com este estudo, podemos citar o de Gadelha et al. (2016), onde realizaram uma análise quantitativa sobre as famílias beneficiarias elegíveis e não elegíveis do Programa Bolsa Família para o ano de 2010, construindo um indicador de focalização baseados em medidas de alcance e precisão. Com este indicador, concluíram que os percentuais associados ao erro tipo I (exclusão indevida) são menores que percentuais associados ao erro tipo II (inclusão indevida), com isso, sugeriram que se houvesse uma realocação dos benefícios concedidos às famílias não elegíveis para famílias elegíveis excluídas, levaria uma universalização do programa.

No estudo de Marconato et al. (2015) estabeleceu-se uma relação entre à taxa de pobreza e o benefício médio do Programa Bolsa Família no ano de 2010 para os municípios de Santa Catarina, com intuito de verificar se o benefício está focalizado em regiões com maiores taxas de pobreza. A metodologia usada consistiu na análise explanatória dos dados espaciais (AEDE), utilizando o coeficiente I de Moran bivariado, gráfico de dispersão de Moran bivariado e o mapa de cluster das variáveis. Pelos resultados, observou-se que os municípios com elevada taxa de pobreza possuem alto valor do benefício médio e municípios com baixo índice de pobreza apresentam baixo valor do benefício médio. Já no estudo de Brambilla et al. (2016), similar ao estudo de Marconato et al. (2015), porém para os municípios do Paraná, nos anos de 2004, 2008 e 2012, os resultados indicaram a existência de

DRd - Desenvolvimento Regional em debate (ISSNe 2237-9029) 
autocorrelação positiva entre o valor médio repassado do Programa Social Bolsa Família e a taxa de pobreza.

Outros estudos focaram na análise espacial da taxa de pobreza, um deles é o de Romero (2016), o qual elaborou uma análise espacial da pobreza para os municípios de Minas Gerais, nos anos 1991 e 2000. A pobreza foi mensurada a partir dos valores do Índice de Desenvolvimento Humano Municipal (IDHM), juntamente com outros determinantes, como IDHM-Educação, IDHM-Longevidade e IDHM-Renda para cada munícipio. A metodologia consistiu na estatística I de Moran local e global. Os resultados do estudo apontaram a existência de dependência espacial da pobreza (medida pelo IDHM) em relação à renda, longevidade e educação, e também encontrou clusters de pobreza, evidenciando que municípios pobres estão rodeados de municípios pobres.

Silva, Borges e Parré (2013), também analisaram a distribuição espacial da taxa de pobreza, para os municípios do estado do Paraná, utilizando modelos econométricos espaciais. Utilizou três variáveis explicativas para justificar a variável dependente taxa de pobreza, sendo índice de GINI, renda média e grau de urbanização. Com os modelos econométricos e as estatísticas I de Moran local e global, os autores encontraram evidências que o aumento da renda média e urbanização provocam diminuição na taxa de pobreza e um aumento do índice de GINI eleva a taxa de pobreza.

\section{METODOLOGIA}

Segundo Almeida (2012), a análise exploratória de dados espaciais é a coleção de técnicas para descrever e visualizar distribuições espaciais, identificar localidades atípicas, descobrir padrões de associação espacial e sugerir diferentes regimes espaciais e outras formas de instabilidade. Ainda conforme Almeida (2012), a AEDE consiste em uma coleção de ferramentas que permite conhecer os dados espaciais e para efetuar a modelagem econométrica espacial. Portanto, a AEDE representa a primeira etapa na investigação de um fenômeno de interesse. As seguintes técnicas para visualizar distribuições espaciais podem ser aplicadas:

\subsection{AUTOCORRELAÇÃO ESPACIAL GLOBAL UNIVARIADA E BIVARIADA}

O primeiro passo no estudo da AEDE é testar hipótese que os dados espaciais sejam distribuídos aleatoriamente, para isso, Moran (1948) propôs a autocorrelação espacial global univariada, com intuito de analisar a autocorrelação espacial de uma variável. Sua expressão é dada por: 


$$
\mathbf{I}=\frac{\mathbf{n}}{\mathbf{s}_{\mathbf{0}}} \sum_{\mathrm{i}} \sum_{\mathbf{j}} \mathbf{w}_{\mathrm{ij}} \mathbf{z}_{\mathbf{i}} \mathbf{z}_{\mathbf{i j}} / \sum_{\mathbf{i}=\mathbf{1}}^{\mathbf{n}} \mathbf{z}_{\mathbf{i}}^{2}
$$

A notação é dada por:

$-n$ é o número de regiões;

$-z$ denota valores da variável de interesse padronizada;

$-W z$ representa valores médios da variável de interesse padronizado nos vizinhos, com uma matriz de ponderação espacial $W$.

A estatística I de Moran para autocorrelação espacial global, é para analisar se os valores de uma variável em uma dada região têm associação, com os valores de outra variável observada em uma região vizinha. A estatística é dada como:

$$
\mathbf{I}^{\mathbf{z}_{1} \mathbf{z}_{2}}=\frac{\mathbf{n}}{\mathbf{S}_{0}} \frac{\mathrm{z}_{1} \mathbf{W z}_{2}}{\mathrm{z}_{1} \mathrm{z}_{2}}
$$

Onde $\mathrm{z}_{1}$ e $\mathrm{z}_{2}$ consistem em duas variáveis diferentes padronizadas.

\subsection{AUTOCORRELAÇÃO ESPACIAL LOCAL UNIVARIADA E BIVARIADA}

Não se deve confiar apenas em estatísticas globais, pois elas podem camuflar padrões locais de autocorrelações espaciais, por isso, surgiu à estatística I de Moran Local univariado, com a capacidade de capturar padrões locais de autocorrelação espacial, denominados indicadores de Lisa (ALMEIDA, 2012). A autocorrelação local univariada e dada pela seguinte expressão:

$$
\mathbf{I}_{\mathbf{i}}=\mathbf{z}_{\mathbf{i}} \sum_{\mathbf{j}=\mathbf{1}}^{\mathbf{i}} \mathbf{W}_{\mathbf{i j}} \mathbf{z}_{\mathbf{j}}
$$

Onde $I_{i}$ abrange os vizinhos da observação $i$, definidos conforme uma matriz de pesos espaciais.

A autocorrelação espacial bivariada surge quando tem duas variáveis de interesse padronizadas, sendo sua fórmula:

$$
I_{i}=z_{1 i} W z_{2 i}(4)
$$

\subsection{DIAGRAMA DE DISPERSÃO I DE MORAN}

Essa análise tem o propósito de demonstrar a defasagem espacial da variável de interesse padronizada no eixo vertical e o valor da variável de interesse padronizada no eixo horizontal. Como explicado por Almeida (2012), o diagrama de dispersão I de Moran é um 
gráfico de dispersão da nuvem de pontos representando regiões. Para obter a regressão estima-se por Mínimos Quadrados Ordinários (MQO) usando a seguinte expressão:

$$
W z=\alpha+\beta z+\varepsilon
$$

Onde:

$-\alpha$ é a constante da regressão;

$-\beta$ é o coeficiente angular;

$-\varepsilon$ é o termo de erro.

O diagrama fornece muitas informações, tais como quadrantes representando quatro tipos de associação linear espacial, sendo Alto-Alto, Baixo-Baixo, Alto-Baixo e Baixo-Alto. $\mathrm{Na}$ Figura 1 tem-se o exemplo do gráfico de dispersão I de Moran.

Figura 1- Gráfico de dispersão I de Moran.

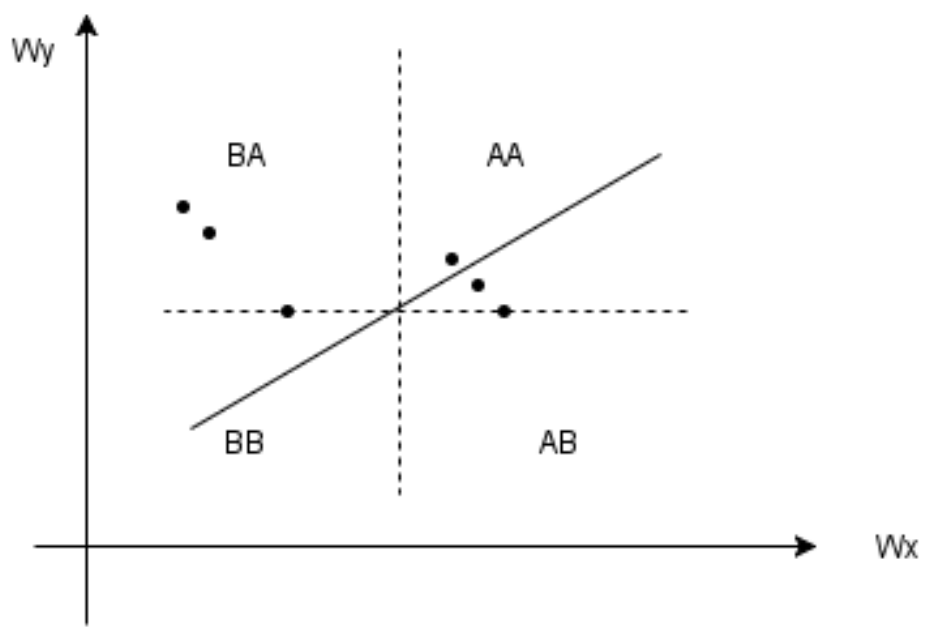

Fonte: Elaboração realizada pelos autores.

\subsection{DETECÇÃO DE OUTLIERS}

Outliers são de duas naturezas: outlier global e outlier espacial. O outlier global é uma observação que foge do restante das observações tanto para cima quanto para baixo. Outliers espaciais são observações discrepantes que não sege mesmo processo de dependência espacial que a maioria dos dados segue (ALMEIDA, 2012).

\subsection{MODELOS DE REGRESSÃO}

Após a AEDE, realiza-se a regressão do modelo. Nesse estudo, foi analisado o ano de 2010, pois este ano realizou-se o Censo, e é possível obter mais informações, como estimativa do número de famílias vivendo em situações de extrema pobreza e pobreza. Quando se tem 
um ano para fazer a análise, há diversas formas de estimações do modelo, contudo o foco será as estimações que levam em consideração a dependência da variável dependente da taxa de cobertura do programa Bolsa Família. Dentre os métodos de estimação com dependência espacial pode citar os seguintes modelos:

i) Modelo SAR apresenta a seguinte expressão:

$$
y=\rho W y+X \beta+\varepsilon(6)
$$

Onde $W y$ é um vetor de defasagem espacial para a variável dependente; $\rho$ coeficiente autorregressivo espacial e $X$ vetor de variáveis explicativas. Segundo Almeida (2012) a estimação é através do método de máxima verossimilhança, sob pressuposto de normalidade assume a seguinte especificação:

$$
\ln L=\frac{-n}{2} \ln \pi-\frac{n}{2} \ln \sigma^{2}+\ln \left|I_{n}-\lambda W\right|-\frac{1}{2 \sigma^{2}}(y-X \beta)^{\prime}\left(I_{n}-\lambda W\right)(y-X \beta)
$$

Após isso, realiza-se as condições de primeira ordem para maximização da função logverossimilhança, para obter as estimativas $\beta$ e $\sigma^{2}$.

ii) Modelo SEM tem-se a seguinte expressão:

$$
\begin{aligned}
& y=X \boldsymbol{\beta}+\xi \\
& \xi=\lambda W \xi+\varepsilon
\end{aligned}
$$

Este modelo apresenta um erro espacial autorregressivo de primeira ordem, sendo $\lambda$ parâmetro do erro autorregressivo espacial que acompanha a defasagem espacial $W \xi$ (Almeida, 2012). A estimação também é através do método de máxima verossimilhança, assumindo a seguinte especificação sob pressuposto de normalidade:

$$
\ln L=\frac{-n}{2} \ln \pi-\frac{n}{2} \ln \sigma^{2}+\ln \left|I_{n}-\lambda W\right|-\frac{1}{2 \sigma^{2}}(y-X \beta)^{\prime\left(I_{n}-\lambda W\right) \prime\left(I_{n}-\lambda W\right)(y-X \beta)}
$$

Realiza-se as condições de primeira ordem para maximização da função log verossimilhança, para obter as estimativas $\beta$ e $\sigma^{2}$.

\subsection{DADOS}

Os dados foram retirados no site do MDS, porém a taxa de pobreza não existe, para fazê-la realizou-se a divisão da estimativa da população em extrema pobreza pela população total. Esta variável tem o intuito de identificar as famílias mais pobres elegíveis para o programa, onde espera-se que quanto maior a taxa de pobreza em uma determinada localidade maior deva ser a taxa de cobertura do programa. O modelo do estudo terá como variável dependente a taxa de cobertura do programa Bolsa Família e as variáveis explicativas são a taxa de pobreza, número de CRAS, valor do programa Bolsa Família repassado para os 
municípios, acompanhamento saúde, acompanhamento educação e Índice de Desenvolvimento Humano.

Os dados são descritos da seguinte maneira:

- cobertura: Refere-se ao percentual de Cobertura do Bolsa Família - perfil Cadastro Único - Cobertura do PBF em relação ao Cadastro Único (\%).

-num_CRAS: Centros de Referência de Assistência Social (CRAS) - Quantidade de CRAS ativos.

-VBF: Valor total repassado do programa para o munícipio.

-pobreza: População em Extrema Pobreza dividido pela população total do município.

-acom_educ: Acompanhamento Educação (Bimestral) - Total de beneficiários acompanhados pela educação (6 a 15 anos).

-acom_sau: Acompanhamento Saúde (Semestral) - Total de famílias acompanhadas pela saúde.

-IDH: Índice de Desenvolvimento Humano (IDH).

\section{RESULTADOS}

\subsection{ESTATÍSTICA DESCRITIVA DAS VARIÁVEIS}

Na Tabela 1 está contida a estatística descritiva do estudo, onde tem-se no total 5.564 municípios e é possível perceber que número de CRAS tem 42 missing. Pela análise verificase que o governo transfere aos beneficiários do programa em média, para cada município, $\mathrm{R} \$ 2.583 .099,00$. E em média cada município tem 2.296 famílias beneficiadas pelo programa.

Tabela 1- Estatística descritiva

\begin{tabular}{c|c|c|c|c|c}
\hline VARIÁVEIS & OBSERVAÇÕES & MÉDIA & DESVIO-PADRÂO & MÍNIMO & MÁXIMO \\
\hline COBERTURA & 5564 & 67,391 & 17,095 & 1,33 & 143,37 \\
\hline POBREZA & 5564 & 13,469 & 12,691 & 0,051 & 66,665 \\
\hline NUM_CRAS & 5522 & 1,496 & 2,005 & 1 & 54 \\
\hline VBF & 5564 & 2593099 & 7170433 & 660 & $2,00 \mathrm{E}+08$ \\
\hline ACOM_SAUDE & 5564 & 1303,86 & 2716,798 & 0 & 64188 \\
\hline ACOM_EDUC & 5564 & 2534,98 & 6916,77 & 2 & 205220 \\
\hline IDH & 5564 & 0,659 & 0,072 & 0,42 & 0.86 \\
\hline
\end{tabular}

Fonte: Elaboração própria a partir de dados do MDS. 
Pode-se observar na Tabela 1 que a média da taxa de pobreza é 13\%. O valor mínimo desta taxa no ano de 2010 foi em torno de $0,05 \%$, e o valor máximo 66,66\%, situados respectivamente nos municípios São Vendelino no Rio Grande do Sul e Marajá do Sena em Maranhão. A média da taxa de cobertura é $67,39 \%$, sendo seu maior valor de $143,37 \%$ localizado em Ubiretama, e menor valor 1,33\%, localizado em Monte Belo do Sul, ambos no estado do Rio Grande do Sul. Esses dados servem de arcabolso para as próximas seções, onde serão feitas análises explanatórias dos dados espaciais e estimações para averiguar se a taxa de cobertura do programa está localizada em municípios que apresentam maior taxa de pobreza.

\subsection{ANÁLISE EXPLORATÓRIA DOS DADOS}

A Figura 2 contêm os mapas de quantile da taxa de cobertura e taxa de pobreza, sendo dividido em três intervalos. Percebe-se que a região do Nordeste há uma grande taxa de pobreza no ano de 2010 e também alta taxa de cobertura, logo nesta região o programa está focalizado. Na região Norte está elevada a taxa de pobreza, porém a taxa de cobertura não segue essa elevação, o que torna necessário elevar o programa nesta região para amenizar a pobreza das famílias. A região Norte é composta pelo segundo estrato no mapa da taxa de cobertura, variando de $60,5 \%$ a $75,8 \%$ e pelo terceiro estrato no mapa da taxa de pobreza, com variação entre $3,88 \%$ a $17,7 \%$.

Figura 2- Mapa de quantile da taxa de cobertura do PBF (a) e taxa de pobreza (b) dos municípios.

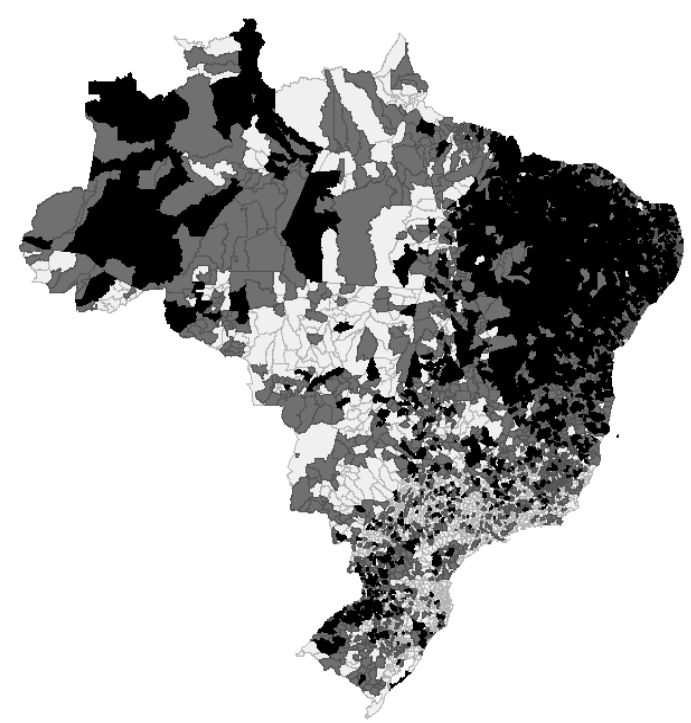

a)

Quantile: COBERTURA

$\square[1.33: 60.4](1855)$

$\square[60.5: 75.8](1854)$

[75.8: 143] (1855) 
b)

Quantile: POBREZA

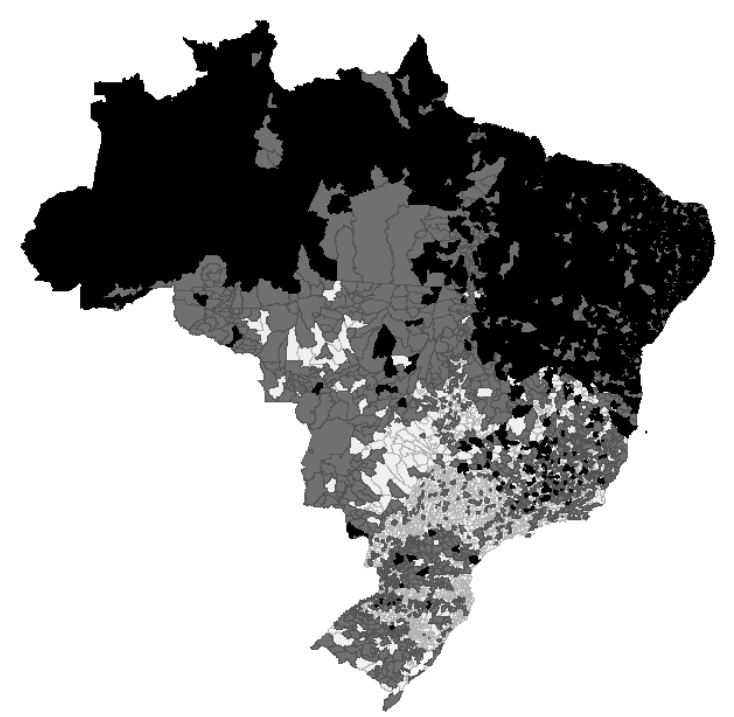

$\square[0.0514: 3.87](1855)$

$\square[3.88: 17.7](1854)$

[17.7: 66.7] (1855)

Fonte: Elaboração própria a partir de dados do MDS, feitos no software Geoda.

$\mathrm{Na}$ Figura $3 \mathrm{~b}$ encontra-se os mapas Box-Map, com hinge 1.5, das taxas de pobreza e cobertura. É possível visualizar nos mapas, que 19 municípios são outliers globais superiores da taxa de pobreza, ou seja, 19 municípios apresentam elevadas taxas de pobrezas em relação aos demais municípios. Estes municípios estão localizados nos estados do Acre, Amazonas, Maranhão, Piauí, Roraima. Na Figura 3a tem-se a taxa de cobertura, na qual 13 municípios são outliers superiores, estando localizados nos estados de Bahia, Maranhão, Pará, Paraná, Pernambuco, Piauí, Rio Grande do Sul, São Paulo. 
Figura 3 - Box-Map da taxa de cobertura do PBF (a) e taxa de pobreza (b) dos municípios

a)

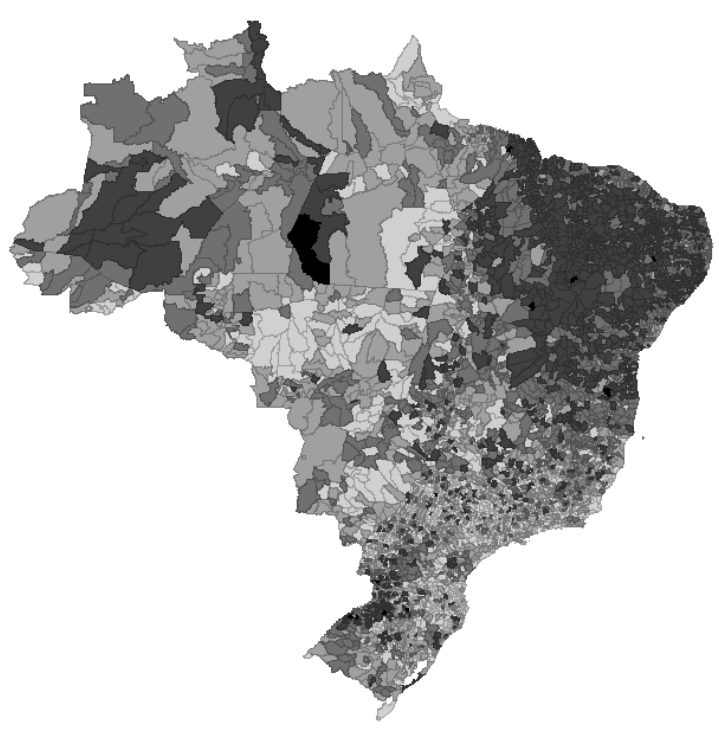

Hinge $=1.5:$ COBERTURA

$\square$ Lower outlier (30) [1.33: 20.1]

$<25 \%$ (1361) [20.1: 55.9]

$25 \%-50 \%$ (1391) [55.9:68.7]

$50 \%-75 \%(1392)[68.7: 79.8]$

$>75 \%(1377)[79.8: 116]$

Upper outlier (13) [116: inf]

b)

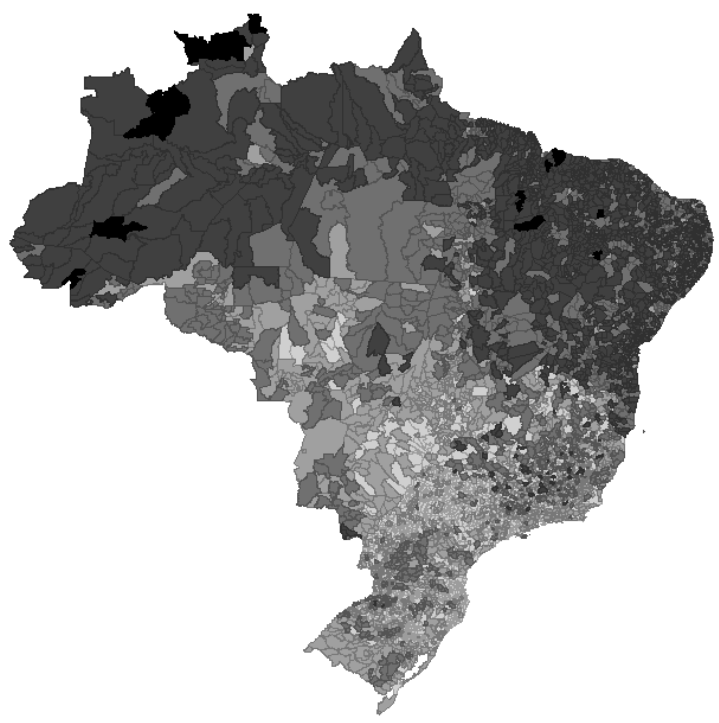

Hinge $=1.5:$ POBREZA

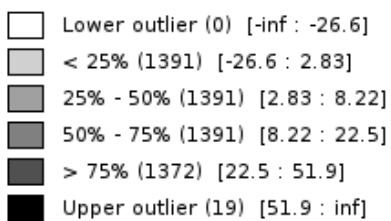

Fonte: Elaboração própria a partir de dados do MDS, feitos no software Geoda.

Para prosseguir a análise, foi necessário definir uma matriz de ponderação espacial para as variáveis taxa de cobertura do programa Bolsa Família e taxa de pobreza. Optou-se

DRd - Desenvolvimento Regional em debate (ISSNe 2237-9029) 
em utilizar a matriz de k-vizinhos, segundo procedimento de Baumont (2004). Na Tabela 2 temos os valores do I de Moran para as variáveis taxa de cobertura e taxa de pobreza, usando $\mathrm{k}$-vizinhos mais próximos variando $1 \leq \mathrm{k} \leq 20$. Pelos valores das estatísticas I de Moran, os 20 vizinhos mais próximos das variáveis taxa de cobertura e taxa de pobreza, possuem o valor da estatística respectivamente 54,47 e 96,13.

Tabela 2- Valores das estatísticas I de Moran dos k-vizinhos mais próximos para as variáveis Cobertura do PBF e taxa de pobreza

\begin{tabular}{c|c|c}
\hline & COBERTURA & POBREZA \\
\hline K-VIZINHOS MAIS PRÓXIMOS & 20 & 31,21 \\
\hline 2 & 25,14 & 40,61 \\
\hline 3 & 30,5 & 58,92 \\
\hline 4 & 34,12 & 65,07 \\
\hline 5 & 37,5 & 66,13 \\
\hline 6 & 40,07 & 70,63 \\
\hline 7 & 40,07 & 75 \\
\hline 8 & 44,18 & 79,16 \\
\hline 9 & 46,09 & 82,75 \\
\hline 10 & 47,5 & 86,11 \\
\hline 11 & 49,17 & 89,48 \\
\hline 12 & 50,82 & 92,7 \\
\hline 13 & 52,79 & 96,13 \\
\hline 14 & 54,47 & 99,36 \\
\hline 15 & 55,85 & 102,25 \\
\hline 16 & 57,28 & 105,24 \\
\hline 17 & 58,73 & 107,7 \\
\hline 18 & 59,93 & 110,54 \\
\hline 19 & 61,31 & 113,36 \\
\hline
\end{tabular}

Fonte: Elaboração própria a partir de dados do MDS, feitos no software Geoda.

Com as matrizes de ponderações espaciais definidas para as variáveis taxa de cobertura e taxa de pobreza, a Figura 4 apresenta as estatísticas I de Moran Local para tais variáveis. Pela Figura 4 percebe-se que ambas estatísticas foram positivas, tanto a taxa de cobertura quanto a taxa de pobreza, apresentando autocorrelação espacial positiva, altos (baixos) valores da taxa de cobertura e taxa de pobreza estão circundados por altos (baixos) valores destas variáveis nas regiões vizinhas. O p-valor da estatística I de Moran com 999 permutações, para a variável cobertura e a variável taxa de pobreza é igual a 0,001 , ou seja, significativos ao nível de 5\%. 
Figura 4 - Estatísticas I de Moran univariadas da taxa de cobertura do PBF (a) e taxa de pobreza (b) dos municípios

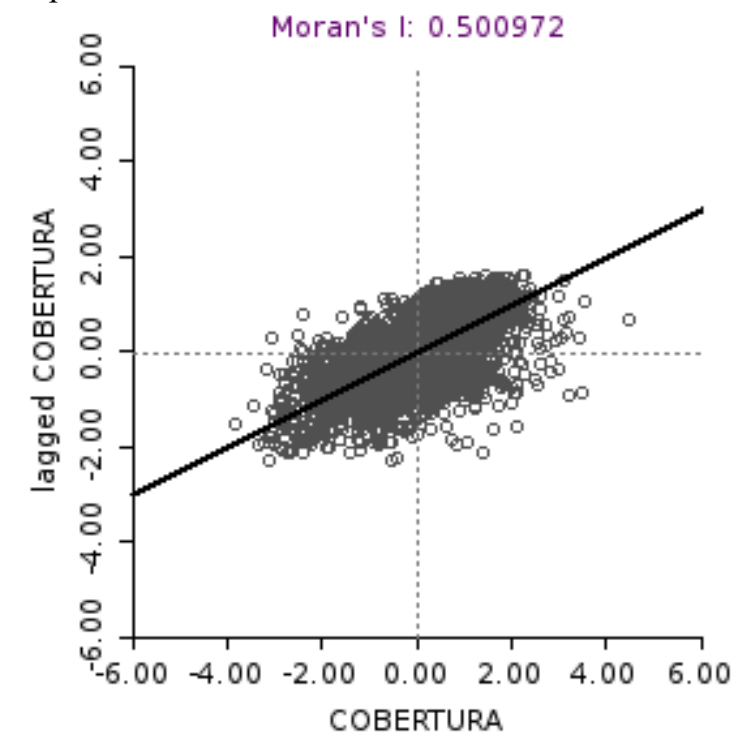

a)

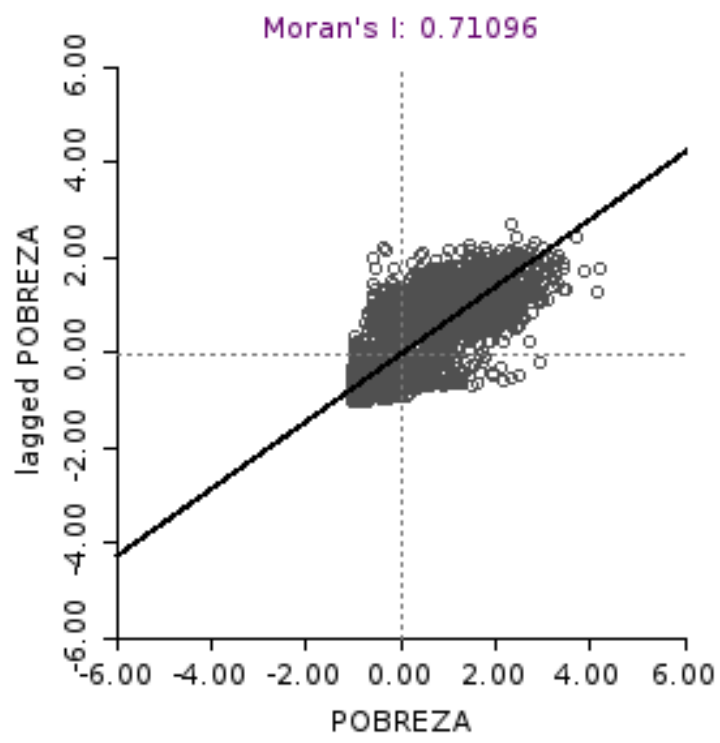

b)

Fonte: Elaboração própria a partir de dados do MDS, feitos no software Geoda.

Na Figura 5 apresenta à estatística I de Moran bivariado, sendo a variável dependente a taxa de cobertura e a explicativa taxa de pobreza. O resultado de 0,49 apontou que as taxas apresentam autocorrelação positiva, ou seja, altos (baixos) valores da taxa de cobertura estão circundados por altos (baixos) valores da variável taxa de pobreza nas regiões vizinhas. $\mathrm{O}$ valor da estatística foi significativo ao nível de 5\%, sendo o $p$-valor igual 0,001. 
Figura 5- Estatísticas I de Moran bivariada da taxa de cobertura do PBF e taxa de pobreza dos municípios

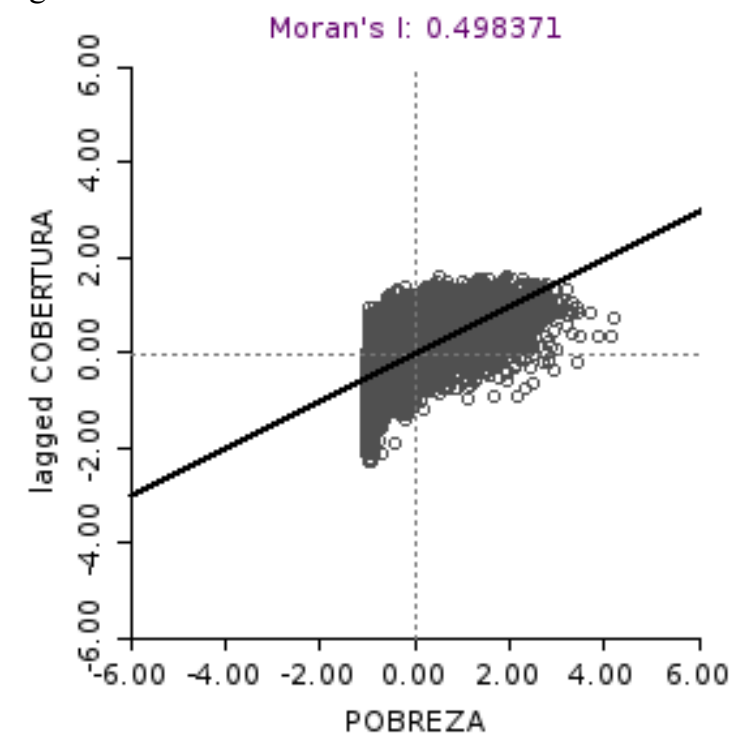

Fonte: Elaboração própria a partir de dados do MDS, feitos no software Geoda.

Nas figuras 6 e 7 estão os mapas de clusters LISA univariados e bivariado da taxa de pobreza e taxa de cobertura. Pela Figura 6, verifica-se no mapa de clusters espaciais da taxa de cobertura as associações alto-alto, baixo-baixo, alto-baixo, baixo-alto da taxa de cobertura. Observando o mapa, um total de 1762 municípios apresentam associação alto-alto, e 1614 municípios apresentam associação baixo-baixo. A maioria dos municípios apresenta altos valores da taxa de cobertura circundados por altos valores desta variável nas regiões vizinhas. 
Figura 6- Mapa de cluster LISA da taxa de cobertura do PBF (a) e da taxa de pobreza (b)

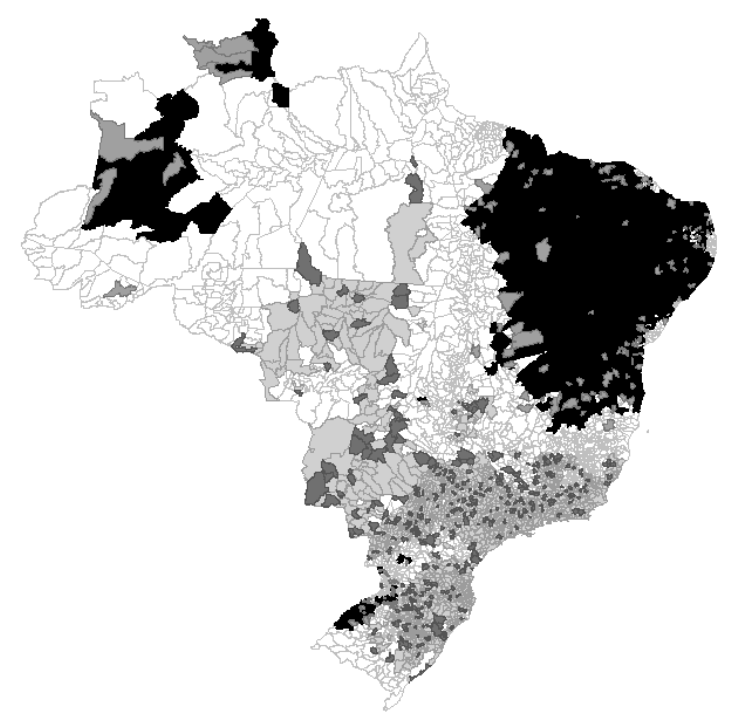

a)

LISA Cluster Map: PESO20, I_COBERTURA

$\square$ Not Significant (1658)

High-High (1762)

Low-Low (1614)

Low-High (155)

High-Low (375)

b)

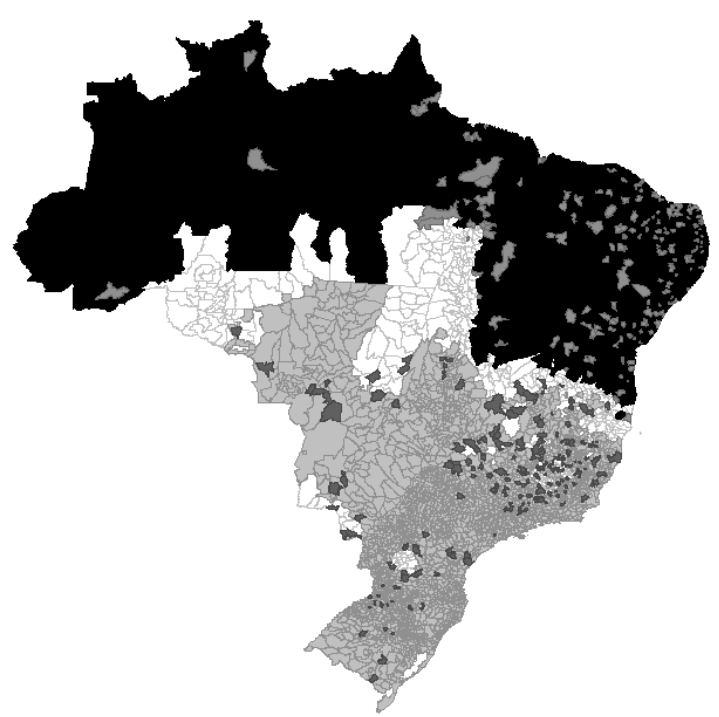

LISA Cluster Map: PESO20, I_POBREZA

Not Significant (443)

High-High (1869)

Low-Low (2856)

Low-High (195)

High-Low (201)

Fonte: Elaboração própria a partir de dados do MDS, feitos no software Geoda.

Na Figura 6 também contém o mapa de cluster LISA da taxa de pobreza. Assim como a taxa de cobertura, padrões locais de autocorrelação espacial positiva, tem-se no total 1869 municípios com associação alto-alto e 2856 municípios com associações baixo-baixo. Ao

DRd - Desenvolvimento Regional em debate (ISSNe 2237-9029)

v. 7, n. 2, p. 118-137, jul./dez. 2017. 
contrário da taxa da cobertura, a maioria dos municípios brasileiros apresentam baixos valores da taxa de pobreza circundados por baixos valores desta variável nas regiões vizinhas.

O mapa de cluster LISA bivariado da taxa de cobertura e da taxa de pobreza estão representados na Figura 7, onde as variáveis apresentam padrões locais de autocorrelação espacial positiva. Pelo mapa de cluster observa-se que a grande parte dos municípios apresenta associação espacial baixa-baixa das taxas de cobertura e pobreza, sendo no total 1883 municípios localizados nas regiões Sul, Sudeste e Centro-Oeste, sendo estas regiões mais ricas economicamente. Analisando associação alto-alto, percebe-se que a maioria dos 1584 municípios estão na região Norte e Nordeste, sendo, portanto, as regiões em que municípios com altas taxas de pobreza são circundados por municípios com alta taxa de cobertura do programa Bolsa Família.

Figura 7- Mapa de cluster LISA bivariado da taxa de pobreza e taxa de cobertura

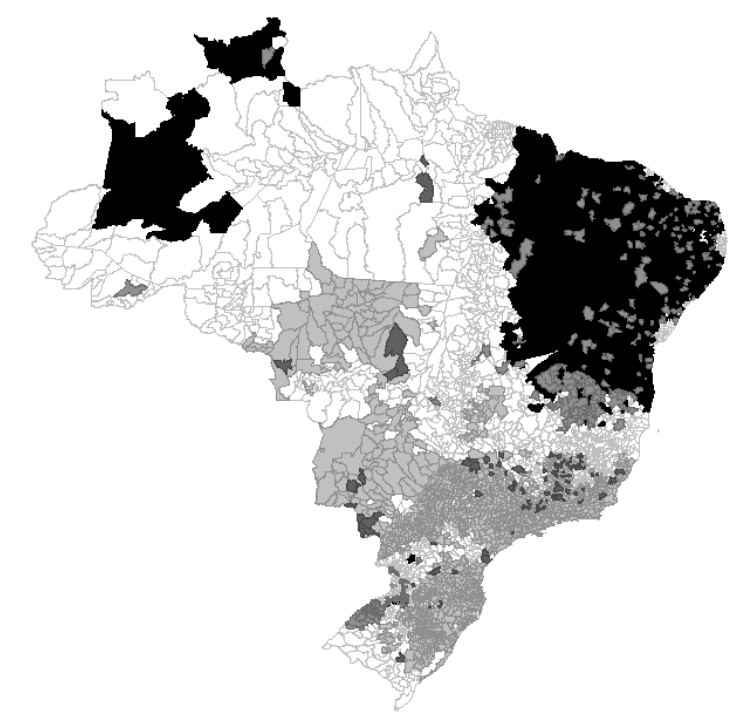

BiLISA Cluster Map: PESO20, POBREZA w/ COBERTURA

Not Significant (1669)

High-High (1584)

Low-Low (1883)

Low-High (328)

High-Low (100)

Fonte: Elaboração própria a partir de dados do MDS, feitos no software Geoda.

\subsection{ESTIMAÇÕES}

Na Tabela 3 contêm os modelos MQO, SAR e SEM para a variável dependente taxa de cobertura do programa Bolsa Família. Pela tabela verifica-se que nos três modelos os sinais das variáveis foram iguais. No modelo SAR, observado na Tabela 3, a variável W_COBERTURA é a defasagem espacial da variável dependente, sendo positiva e significativa ao nível de significância de $1 \%$. Este modelo informa que a variável taxa de cobertura é influencia pela taxa de cobertura das regiões vizinhas. No modelo SEM tem o $\angle A M B D A$ que acompanha a defasagem $\mathrm{W} \xi$, sendo significativo ao nível de $1 \%$ e com sinal positivo, mostra que os erros estão associados com a média dos erros das regiões vizinhas. 
A escolha do modelo é feita a partir dos critérios Akaike e Schwarz, onde quanto menor os valores dos critérios, melhor é o modelo. O modelo SAR é o modelo mais adequado pois contêm menores valores dos critérios Akaike e Schwarz, sendo respectivamente 43794,3 e 43847,3 .

Os valores estimados no modelo SAR indicaram que a cada $1 \%$ de aumento na taxa de pobreza, a taxa de cobertura aumenta $0,046 \%$, isso significa que a taxa de cobertura do programa demora aumentar em regiões em que a pobreza aumenta mais rapidamente. Nos mapas percebe-se que na região Norte do país, os municípios estão com taxas de pobrezas concentradas, porém a taxa de cobertura está aumentando lentamente nestas regiões. $\mathrm{O}$ programa está bem focalizado na região Nordeste do país, mas não está focalizado na região Norte.

A variável VBF e acom_sau tiveram sinais dos coeficientes positivos, isto significa que quanto maior o valor do benefício repassado pelo governo aos municípios, maior a taxa de cobertura. Se no município houver alto acompanhamento da saúde das famílias cadastradas no programa, a cobertura do programa se torna maior. O acompanhamento da saúde engloba acompanhamento da saúde das gestantes das famílias beneficiadas pelo programa, sendo parte do público-alvo do programa Bolsa Família.

As variáveis IDH, num cras e acom educ tiveram sinais dos coeficientes negativos. Quanto maior o IDH, menor a taxa de cobertura, pois municípios mais desenvolvidos apresentam maior IDH e maiores arrecadações de impostos, não necessitando dos repasses do governo federal.

Elevadas taxas de cobertura do programa estão circundados por regiões com elevadas taxas da mesma variável, isto pode ser visto pela variável dependente defasada espacialmente, W_COBERTURA, sendo o valor do seu coeficiente igual $0,503 \%$, isto significa que alto (baixo) valor da taxa de cobertura nos municípios vizinhos aumenta (diminui) o valor da taxa de cobertura para um dado município.

Tabela 3- Modelo MQO, SAR e SEM para a variável dependente taxa de Cobertura do Programa Bolsa Família

\begin{tabular}{|c|c|c|c|c|c|c|c|c|}
\hline \multicolumn{9}{|c|}{ VARIÁVEL DEPENDENTE COBERTURA } \\
\hline \multicolumn{3}{|c|}{ MQO } & \multicolumn{3}{|c|}{ SAR } & \multicolumn{3}{|c|}{ SEM } \\
\hline $\begin{array}{c}\text { VARIÁVEIS } \\
\text { EXPLICATIVAS }\end{array}$ & COEFICIENTES & $\begin{array}{l}\text { DESVIO- } \\
\text { PADRÃO }\end{array}$ & $\begin{array}{c}\text { VARIÁVEIS } \\
\text { EXPLICATIVAS }\end{array}$ & COEFICIENTES & $\begin{array}{l}\text { DESVIO- } \\
\text { PADRÃO }\end{array}$ & $\begin{array}{c}\text { VARIÁVEIS } \\
\text { EXPLICATIVAS }\end{array}$ & COEFICIENTES & $\begin{array}{l}\text { DESVIO- } \\
\text { PADRÃO }\end{array}$ \\
\hline & & & W_COBERTURA & 0,503 & 0,014 & LAMBDA & 0,5147 & 0,0159 \\
\hline CONSTANTE & 136,21 & 2,84 & CONSTANTE & 73,13 & 3,13 & CONSTANTE & 126,56 & 3,08 \\
\hline NUM_CRAS & $-0,54$ & 0,17 & NUM_CRAS & $-0,38$ & 0,15 & NUM_CRAS & $-0,55$ & 0,157 \\
\hline IDH & $-108,1$ & & IDH & $-61,78$ & 3,82 & IDH & $-91,97$ & 4,44 \\
\hline POBREZA & 0,172 & 0,02 & POBREZA & 0,046 & 0,02 & POBREZA & 0,107 & 0,02 \\
\hline ACOM_SAU & 0,0013 & 0,00016 & ACOM SAU & 0,0007 & 0,0001 & ACOM SAU & 0,001 & 0,0001 \\
\hline ACOM_EDU & $-0,0012$ & 0,00013 & ACOM_EDU & $-0,0009$ & 0,0001 & ACOM_EDU & $-0,0007$ & 0,0001 \\
\hline $\mathrm{VBF}$ & $9,22 \mathrm{E}-07$ & 1,11E-07 & $\mathrm{VBF}$ & $7,68 \mathrm{E}-07$ & 9,94E-08 & $\mathrm{VBF}$ & 5,63E-07 & $1,01 \mathrm{E}-08$ \\
\hline $\mathrm{R}^{2}$ & 0,372 & & $\mathrm{R}^{2}$ & 0,502 & & $\mathrm{R}^{2}$ & 0,485 & \\
\hline AKAIKE & 44796,9 & & AKAIKE & 43794,3 & & AKAIKE & 43991,4 & \\
\hline SCHWARZ & 44843,3 & & SCHWARZ & 43847,3 & & SCHWARZ & 44037,7 & \\
\hline
\end{tabular}

Fonte: Elaboração própria a partir de dados do MDS, feitos no software Geoda. 


\section{CONSIDERAÇÕES FINAIS}

O principal objetivo do programa Bolsa Família é contribuir para superação da pobreza através da transferência de renda direta as famílias. O programa é considerado focalizado se atender famílias que vivem em situação de extrema pobreza e pobreza, constituindo o público-alvo do programa, sendo que a identificação destas famílias é feita pelo Cadastro Único. Este trabalho analisou se a cobertura do programa Bolsa Família está elevada em municípios que apresentam altas taxas de pobreza no ano de 2010.

Nesse estudo utilizou-se AEDE e estimação pelos modelos MQO, SAR e SEM. Pela AEDE, observou que grande parte dos municípios que apresentam associações espaciais baixas-baixas das taxas de cobertura e pobreza, está localizada nas regiões Sul, Sudeste e Centro-Oeste. Analisando os mapas quantile, nos quais apresentam as associações, percebe-se que na região Nordeste têm-se fortemente a associação alto-alto, sendo, portanto, região em que municípios com altas taxas de pobreza circundadas por municípios com alta taxa de cobertura do programa Bolsa Família.

Todos os modelos econométricos confirmaram os resultados do I de Moran univariado e bivariado. No modelo SAR é possível constatar que a cada $1 \%$ de aumento na taxa de pobreza a taxa de cobertura aumenta $0,046 \%$. Isso significa que a taxa de cobertura do programa demora aumentar em regiões em que a pobreza aumenta mais rapidamente.

Verificam-se também pelos resultados, que pelos estratos do mapa quantile, a região Norte apresenta elevadas taxas de pobreza, porém as taxas de cobertura não são elevadas como esperado.

\section{REFERÊNCIAS}

ALMEIDA, Eduardo. Econometria espacial. Campinas (SP): Alínea, 2012.

BARROS, R. P. et al. A focalização do Programa Bolsa Família em perspectiva comparada. In: IPEA. Bolsa Família 2003-2010: avanços e desafios. Brasília: Governo Federal, 2010. v. 2

BAUMONT, C.; ERTUR, C.; GALLO, J. Spatial analysis of employment and population density: the case of the agglomeration of Dijon 1999. Geographical analysis, v. 36, n. 2, p. 146-176, 2004.

BRAMBILLA, M. A. et al. Programa Bolsa Família: Uma Análise Espacial nos Municípios do Paraná em 2004, 2008 e 2012. Revista de Economia, v. 40, a. 38, n. 3, p. 47-67, set./dez./2014. 
CAMARGO, C. F. et al. Perfil socioeconômico dos beneficiários do programa Bolsa Família. In: IPEA. Programa Bolsa Família: uma década de inclusão e cidadania. Brasília: Governo Federal, 2013.

GADELHA, S. R. D. B et al. Uma investigação sobre a focalização do programa Bolsa Família e seus determinantes imediatos. In: ENCONTRO DA ASSOCIAÇÃO NACIONAL DOS CENTROS DE PÓS-GRADUAÇÃO EM ECONOMIA; 43. Foz do Iguaçu, PR Anais..., Foz do Iguaçu: ANPEC, 2016.

KERSTENETZKY, C. L. Redistribuição e desenvolvimento? A economia política do programa bolsa família. Dados-Revista de Ciências Sociais, v. 52, n. 1, 2009, p. 53-83.

MARCONATO, M. et al. A focalização do Programa Bolsa Família nos municípios de Santa Catarina: uma análise espacial. Informe Gepec, v. 19, n. 1, p. 38-54, 2015.

MORAN, P. A. P. The interpretation of statistical maps. Journal of the Royal Statistical Society. Series B (Methodological), v. 10, n. 2, p.243-251 1948.

ROMERO, J.A.R. Analise espacial da pobreza municipal no estado de minas gerais-19912000. In: ENCONTRO NACIONAL DE ESTUDOS POPULACIONAIS (ABEP); 14. Caxambú, 2006. Anais..., Caxambú, MG, 2006.

SILVA, L. N. S.; BORGES, M. J.; PARRÉ, J. L. Distribuição espacial da pobreza no Paraná. Revista de Economia, v. 39, a. 37, n. 3, set./dez. 2014, p. 35-58.

UNIÃO, Controladoria Geral da. Olho vivo no dinheiro público: um guia para os cidadãos garantir os seus direitos. 2 ed. 2009. Disponível em: <http://www.avozdocidadao.com.br/ images/cartillha_cgu_baixa.pdf $>$. Acessado em: 28 ago.2017.

VUOLO, R. M. L. Los programas de transferencias monetarias condicionadas en América Latina y las perspectivas de la renta básica o ingreso ciudadano: un análisis en base a los programas" Bolsa família" de Brasil y" Asignación universal por hijo para protección social". Revista internacional de pensamiento político, v. 6, p. 193-222, 2016.

Artigo recebido em: 25/08/2017

Artigo aprovado em: 08/10/2017 